\title{
RESEARCH
}

\section{DEPRESSION AND ANXIETY LEVELS IN A GROUP OF ELDERLY WITH TEMPOROMANDIBULAR DISORDERS}

Turkish Journal of Geriatrics DOI: 10.31086/tigeri.2021.237 2021; 24(3): 397-406

- Ahmet Taylan ÇEBi ${ }^{1}$

- Nihat YILMAZ2

- Fatih KARAYÜREK ${ }^{3}$

- Aydın GÜLSES ${ }^{4}$
CORRESPONDANCE

\section{${ }^{3}$ Fatih KARAYÜREK}

Çankırı Karatekin University, Department of Periodontology, Çankırl, Turkey

Phone: +905534067526

e-mail: fatihkarayurek@karatekin.edu.tr

Received: May 31, 2021

Accepted: Aug 22, 2021

${ }^{1}$ Karabük University, Department of Oral and Maxillofacial Surgery, Karabük, Turkey

${ }^{2}$ Karabük University, Department of Otorhinolaryngology, Karabük, Turkey

${ }^{3}$ Çankırı Karatekin University, Department of Periodontology, Çankırı, Turkey

${ }^{4}$ Christian Albrechts University, Department of Oral and Maxillofacial Surgery, Kiel, Almanya

\begin{abstract}
Introduction: The objective of this study was to assess stress and anxiety in a sample of elderly patients suffering from temporomandibular joint disorders who did not have previously diagnosed emotional disturbances. It is well known that patients presenting with temporomandibular joint disorders are more commonly affected by anxiety and depression. However, there is a lack of data regarding the prevalence of undiagnosed anxiety and emotional stress disorders among elderly patients with temporomandibular joint disorders.
\end{abstract}

Materials and Method: Clinical examination was performed using Research Diagnostic Criteria (Axis I). Patients with previously diagnosed and managed psychiatric and psychological disorders were excluded. Magnetic resonance imaging findings were also used to confirm the diagnosis. Beck Depression Inventory and Beck Anxiety Inventory forms were obtained to assess stress and anxiety levels.

Results: A total of 125 patients (103 [82.4\%] women and 22 [17.6\%] men) aged 60 to $87(71.89 \pm 10.39$ ) years were included. The highest depression and anxiety scores were found among patients suffering from bruxism, followed by those with disc displacement without reduction and myofascial pain syndrome. All the patients showed significantly elevated depression levels.

Conclusion: The emotional health status of elderly patients with intraand extra-articular temporomandibular joint disorders should be attentively assessed.

Key words: Anxiety; Depression; Temporomandibular Joint Disorder. 


\section{INTRODUCTION}

Temporomandibular joint disorders (TMDs) are fundamentally painful, affecting the temporomandibular joint (TMJ) together with the masticatory system and innervation of the head and neck (1), and they are detected at an incidence rate of about $25 \%$ in general society (1). Considering the complex modality of the TMJ, the symptoms can be variable, including local or spreading pain in the TMJ or masticatory muscles, crepitation, dislocation, or clicking on the movement of the TMJ, severe headache in the temporal region, and tinnitus (2). Even though TMD symptoms were seen in each age group and affect $90 \%$ of the general population at one or another life stage (3), TMDs were mostly determined between the third and fifth decades of life (4). However, studies focusing on TMDs in elderly patients are limited (5).

The aetiological factors of TMDs could be biological, environmental, social, emotional, and/or cognitive. Moreover, the effects of emotional status on the symptoms and prognosis of TMDs have been widely discussed and several studies have also shown that parafunctional habits, muscle hyperactivity, and micro-occlusal trauma could result from emotional stress disorders; thus, elevated stress, anxiety, and/or depression levels could correlate with TMD-related signs and symptoms (6).

The classification of TMDs was described at the International Research Diagnostic Criteria for Temporomandibular Dysfunction Consortium Network in 2013 (7) the Validation Project determined that the RDC/TMD Axis I validity was below the target sensitivity of $\geq 0.70$ and specificity of $\geq 0.95$. Consequently, these empirical results supported the development of revised RDC/TMD Axis I diagnostic algorithms that were subsequently demonstrated to be valid for the most common pain-related TMD and for one temporomandibular joint (TMJ. TMDs were mainly subdivided into intra- and ex- tra-articular pathologies. Among extra-articular manifestations, myofascial pain syndrome has been shown to be the most frequent cause of orofacial pain $(42.0 \%)$, whereas disc displacement without reduction (32.1\%) is the most common painful intra-articular pathology (7)the Validation Project determined that the RDC/TMD Axis I validity was below the target sensitivity of $\geq 0.70$ and specificity of $\geq 0.95$. Consequently, these empirical results supported the development of revised RDC/TMD Axis I diagnostic algorithms that were subsequently demonstrated to be valid for the most common pain-related TMD and for one temporomandibular joint (TMJ. Besides, osteoarthrosis is suggested to be the magnetic resonance characteristic of TMJ disc displacement in elderly patients (5).

Over the last decades, the correlation between TMDs and emotional status has been widely studied $(6,8)$. It is well known that patients presenting with TMDs are more commonly affected by anxiety and depression (6). However, there seems to be a lack of data on the prevalence of undiagnosed anxiety and emotional stress disorders among elderly patients with TMDs. Therefore, the objective of this study was to assess the level and prevalence of stress and anxiety in a sample of patients suffering from TMDs.

\section{MATERIALS AND METHODS}

This study was conducted in accordance with the Helsinki Declaration. Ethical approval was obtained from the Committee of Non-invasive Clinical Studies (03 January 2018, 1/17). The patients were informed, and written consent forms were obtained.

\section{Study Design}

This study was conducted between April and December 2020; the study groups consisted of patients who were referred to the Department of Oral 
and Maxillofacial Surgery with complaints of TMDs. The Research Diagnostic Criteria for TMDs (RDC/ TMD) Axis I was applied to identify TMDs (7)the Validation Project determined that the RDC/TMD Axis I validity was below the target sensitivity of $\geq$ 0.70 and specificity of $\geq 0.95$. Consequently, these empirical results supported the development of revised RDC/TMD Axis I diagnostic algorithms that were subsequently demonstrated to be valid for the most common pain-related TMD and for one temporomandibular joint (TMJ. According to The Research Diagnostic Criteria for TMDs (RDC/TMD) Axis I (7);

\section{Group I: Muscle Disorders}

- I. a. Myofascial Pain

- I. b. Myofascial Pain with Limited Opening

- I. c. Myofascial Pain with Referral

- I. d. Temporalis Tendonitis

Group II: Disc Displacements

- II. a. Disc Displacement with Reduction

- II. b. Disc Displacement without Reduction without Limited Opening

- II. c. Disc Internal Derangement with Reduction with Transient Limited Opening

- II. d. Disc Internal Derangement without Reduction with Limited Opening

Group III: Arthralgia/Arthritis/Arthrosis

- III. a. Arthralgia /Arthritis

- III. b. Osteoarthritis /Degenerative Joint Disease

- III. c. Osteoarthrosis /Degenerative Joint Disease

Group IV: Temporomandibular Joint Hypermobility

- IV. a. Subluxation/Luxation

Group V: Tension-type Headache with Temporalis Muscle Tenderness

- V. a. Infrequent Episodic Tension-type Headache Involving the Temporalis Muscle
- V. b. Frequent Episodic Tension-type Headache Involving the Temporalis Muscle

- V. c. Chronic Tension-type Headache Involving the Temporalis Muscle

Magnetic resonance imaging (MRI) findings were also used to confirm the diagnosis. The patients were subdivided into five groups according to their diagnosis:

- Group I: Anterior disc displacement with reduction

- Group II: Anterior disc displacement without reduction

- Group III: Bruxism

- Group IV: Myofascial pain syndrome

- Group V: Control group (patients who presented with complaints other than TMDs)

Patients with previously diagnosed and managed psychiatric and psychological disorders such as depression, anxiety, and personality disorders, neurological disorders, Eagle syndrome, rheumatologic disorders affecting the TMJ, and acute TMD symptoms and those younger than 60 years were excluded. Their socio-demographic data and Beck Depression Inventory (BDI) and Beck Anxiety Inventory (BAl) forms were obtained.

Briefly, the BDI consists of 21 questions quantified by summing the scores between 0 and 3 obtained for each answer, which defines the depression level (9). Values 0-9 are determined as normal, 10-18 mild, 19-29 moderate, and 30-63 severe depression. Similarly, the BAI is a Likert-type scale scored between 0 and 3 , which consists of 21 items and is used to determine the frequency and level of anxiety symptoms (10). Values $0-7$ are considered normal, 8-15 mild, 16-25 moderate, and 26-63 severe anxiety.

According to the answers and scores obtained from the forms and scales, the relationship determinants of anxiety and depression and parameters such as the classification of TMDs into two groups 
and their age and gender distributions were comparatively assessed.

\section{Statistical Analysis}

Study data were analysed using Minitab 17 (Minitab Inc., State College, PA, USA). The ShapiroWilk test was run to analyse the normality of the parameters. In descriptive statistical evaluation, one-way analysis of variance, chi-squared test, and Bonferroni corrections were used to compare the groups. The Mann-Whitney U test was used to evaluate parameters which did not show normal distribution. Statistical results were evaluated at a $95 \%$ confidence interval and $p<0.001$ and $<0.05$ significance level.

\section{RESULTS}

A total of 125 patients (103 [82.4\%] women and 22 [17.6\%] men) aged 60-87 (71.89 \pm 10.39$)$ years were included. The statistical differences in terms of age $(p=0.141,>0.05$ ) were insignificant (Table
1). The mean age of the patients with anterior disc displacement with reduction was higher than that of the other groups (Table 1). There was no statistically significant difference in terms of the gender distribution among the subgroups ( $p=0.143,>0.05$; Table 1). However, the number of women was significantly higher in total.

A statistically significant difference was seen in depression scores between the patients with TMDs and the control group $\left(p=0.000^{*},<0.001\right.$; Table 2). The highest depression scores were noted in the bruxism and disc displacement without reduction subgroups ( $p=0.000^{*}$; Table 3 ). A statistically significant difference was also observed in the assessment of anxiety scores between the groups ( $p$ $\left.=0.000^{*},<0.001\right)$. The highest anxiety scores were found in the bruxism and myofascial pain syndrome groups (Table 3). However, a comparative analysis of the subgroups revealed that the patients with bruxism and disc displacement without reduction showed the highest differences compared to the control group, and both differences were also nearly equal (Table 4).

Table 1. Evaluation of demographic data between subgroups.

\begin{tabular}{|l|c|c|c|}
\hline \multicolumn{1}{|c|}{ Classification of TMD } & Age & \multicolumn{2}{c|}{ Gender } \\
\cline { 2 - 4 } & $(n)$ & 21 \\
\hline Disc Displacement with Reduction & $67.30 \pm 6.90$ & 20 & 5 \\
\hline Disc Displacement without Reduction & $71.88 \pm 11.77$ & 21 & 5 \\
\hline Bruxism & $73.85 \pm 12.32$ & 20 & 5 \\
\hline Myofascial Pain Syndrome & $74.93 \pm 11.96$ & 21 & 4 \\
\hline Control & $69.25 \pm 7.71$ & $2 p=0.143$ \\
\hline$p$ & $1 p=0.141$ & & 5 \\
\hline
\end{tabular}

The number of women was relatively higher in all groups. (TMD: temporomandibular disorder) 
Table 2. Evaluation of depression scores according to the Beck depression inventory.

\begin{tabular}{|l|c|c|}
\hline \multicolumn{1}{|c|}{ Classification of TMD } & BDS & Evaluation of BDS \\
\hline Disc Displacement with Reduction & $9.75 \pm 7.70$ & Mild depression \\
\hline Disc Displacement without Reduction & $12.40 \pm 7.90$ & Mild depression \\
\hline Bruxism & $16.60 \pm 12.00$ & Mild depression \\
\hline Myofascial Pain Syndrome & $11.28 \pm 8.000^{\star}$ \\
\hline Control & $5.675 \pm 3.892$ & Mild depression \\
\hline
\end{tabular}

All patients showed mild depression scores, which were also significantly higher compared to the control group. (TMD: temporomandibular disorder, BDS: Beck Depression Score)

Table 3. Comparison of Beck Depression Scores between subgroups.

\begin{tabular}{|c|c|}
\hline Comparison of Beck Depression Scores & p \\
\hline Disc Displacement with vs without Reduction & 0.079 \\
\hline Bruxism vs Disc Displacement with Reduction & $0.011^{*}$ \\
\hline Disc Displacement with Reduction vs Myofascial Pain Syndrome & 0.511 \\
\hline Disc Displacement with Reduction vs Control & $0.020^{*}$ \\
\hline Disc Displacement without Reduction vs Bruxism & 0.188 \\
\hline Disc Displacement without Reduction vs Myofascial Pain Syndrome & 0.625 \\
\hline Disc Displacement without Reduction vs Control & $0.000^{*}$ \\
\hline Bruxism vs Myofascial Pain Syndrome & $0.042^{*}$ \\
\hline Bruxism vs Control & $0.000^{*}$ \\
\hline Myofascial Pain Syndrome vs Control & $0.007 *$ \\
\hline
\end{tabular}

"*" showing the significant differences. All subgroups showed significantly higher values compared to the control group. Patients with Bruxism and disc displacement without reduction showed the highest values, followed by the myofascial pain syndrome. 
Table 4. Evaluation of anxiety scores between subgroups according to the Beck anxiety inventory.

\begin{tabular}{|l|c|c|}
\hline \multicolumn{1}{|c|}{ Classification of TMD } & BAS & Evaluation of BAS \\
\hline Disc Displacement with Reduction & $11.28 \pm 9.74$ & Mild anxiety \\
\hline Disc Displacement without Reduction & $14.03 \pm 8.71$ & Mild anxiety \\
\hline Bruxism & $22.65 \pm 13.50$ & Moderate anxiety \\
\hline Myofascial Pain Syndrome & $16.23 \pm 14.70$ & Moderate anxiety \\
\hline Control & $6.000 *$ & Normal \\
\hline
\end{tabular}

Elevated anxiety scores were observed in all subgroup, whereas the patients with disc displacements showed mild and bruxism and myofascial pain syndrome patients showed moderate anxiety scores respectively. (TMD: temporomandibular disorder, BAS: Beck Anxiety Score)

Table 5. Comparison of Beck Anxiety Scores between subgroups.

\begin{tabular}{|l|c|}
\hline Comparison of Beck Anxiety Scores & p \\
\hline Disc Displacement with vs without Reduction & 0.091 \\
\hline Bruxism vs Disc Displacement with Reduction & $0.002^{\star}$ \\
\hline Disc Displacement with Reduction vs Myofascial Pain Syndrome & $0.021^{\star}$ \\
\hline Disc Displacement with Reduction vs Control & $0.004^{\star}$ \\
\hline Disc Displacement without Reduction vs Bruxism & 0.699 \\
\hline Disc Displacement without Reduction vs Myofascial Pain Syndrome & $0.000^{\star}$ \\
\hline Disc Displacement without Reduction vs Control & $0.039^{\star}$ \\
\hline Bruxism vs Myofascial Pain Syndrome & $0.000^{\star}$ \\
\hline Bruxism vs Control & $0.001^{*}$ \\
\hline Myofascial Pain Syndrome vs Control & \\
\hline
\end{tabular}

"*" showing the significant differences. All subgroups showed significantly higher values compared to the control group. Patients with Bruxism and disc displacement without reduction showed the highest differences compared to the control group and both differences were also nearly equal. 
All the TMD subgroups showed significantly higher depression and anxiety levels compared to the control group. The depression and anxiety scores among the subgroups revealed that the patients with bruxism had the highest scores. Those with bruxism and disc displacement without reduction showed the highest differences compared to the control group in the assessment of both depression and anxiety levels $\left(p=0.000^{\star}\right)$.

\section{DISCUSSION}

The role of psychological factors in the aetiology of TMDs cannot be ignored, as they play a great role in many painful conditions and parafunctional habits of the human body (11). Psychological disorders have also been defined as key factors in the development of TMDs by some authors (12). Therefore, the prevalence of anxiety and stress in patients with TMDs has been studied in the literature (13). However, most studies have only focused on the prevalence of psychological disorders among patients with TMDs. The strength of this research, this is the first study to focus on undiagnosed stress and anxiety disturbances in elderly patients with TMDs, our knowledge.

Saeed and Riaz (14)TMD symptoms may be caused by different physiological and/or psychosocial factors. The purpose of this study is to determine the level of depression in patients with TMD. These findings can guide the diagnosis, prevention, and treatment of TMDs. In a prospective study, participants were screened and underwent a baseline physical examination of the head and neck, conducted according to the protocol of Research Diagnostic Criteria Axis I for Temporomandibular Disorders(RDC/TMD tried to determine the depression levels in patients with TMDs and stated that moderate depression was more frequent in these patients (i.e. $30.7 \%)$, followed by severe depression (22.0\%), extreme depression (16.0\%), and borderline clinical depression (10.0\%). Bertoli and de Leeuw (15) reported elevated suicidal ideation, depression, and anxiety levels among patients with TMDs, especially in those with chronic muscle pain, compared to the general population. The current study results showed that patients with intra- and extra-articular TMJ pathologies presented with mild depression levels. Additionally, the assessment of anxiety levels revealed that the patients with intra-articular pathologies showed mild elevated anxiety levels, whereas those with extra-articular pathologies such as bruxism and myofascial pain syndrome showed moderate levels. Generally, both elevated stress and anxiety levels were in accordance with the existing literature (16).

Surprisingly, a comparative analysis of both the anxiety and depression scores of patients suffering from TMDs with disc displacement without reduction and the control group revealed nearly equal differences with the bruxism group. In the literature, an association between disc displacement with reduction and quality of life has been stated previously (17)43 were included in the present study. Fonseca's anamnestic index was used for initial screening while axis I of the Research Diagnostic Criteria for Temporomandibular Disorders (RDC-TMD. However, studies focusing on the relation between TMJ disc displacement without reduction and elevated anxiety and/or depression levels are limited in terms of case studies (18). Considering the prevalence of the condition, this phenomenon might warrant further research.

Ogura et al (5) evaluated the magnetic resonance characteristics of TMJ disc displacement in elderly patients and stated that the incidence of disc displacement with osteoarthrosis was significantly high (41.9\%). In the current study, to avoid undiagnosed rheumatologic conditions which could mimic TMD symptoms and obscure the perceptions of the patients, those with osteoarthrosis of the TMJ were excluded from the study.

The $\mathrm{BDI}$ and $\mathrm{BAl}$ scales were developed by Beck et al (9) and are used to quantify depression and determine the frequency and level of anxiety 
symptoms (10). In the literature, the validity of both methods has been approved by several studies $(19,20)$ pain threshold at pressure, pain vigilance, oral health-related quality of life (OHRQoL. In a recent study, Maślak-Bereś et al (20)as described by the Beck's Depression Inventory (BDI have assessed the relationships between TMD/RDC clinical diagnoses and psycho-emotional status via the BDI and Perceived Stress Scale (PSS-10) and observed that in those with pain, the mean values on the BDI and PSS-10 scales were higher than those pain-free. Because of the complex modality of the TMJ region, MRI is the gold standard for assessing the internal derangements of the TMJ. Therefore, the results of TMD studies based solely on RDC and clinical findings could be controversial. In the current study, the intra-articular derangements of the TMJ were also confirmed via MRI.

Another point to discuss is the similarities in the need for treatment of both TMD and psychological disturbances. TMD-related problems occur in $90 \%$ of the population at least once in a lifetime; however, the need for treatment depends mainly on the pain perception and/or functional disturbances of a patient. Similarly, undiagnosed depression or elevated stress levels are common mental disorders with a prevalence varying from $20 \%$ to $53 \%$ in different decades of life (21), which could have a considerable effect on the health-related quality of life and satisfaction levels of patients. Additionally, it has also been proclaimed that there could be a significant delay in seeking treatment for the mental health of patients with TMD symptoms (22). Like patients with TMDs, not all elderly patients with undiagnosed depression or elevated stress levels need psychological evaluation and management; however, it should be kept in mind that undiagnosed depression could also lead to several outcomes if not managed properly.

It is also well known that because of the ongoing COVID-19 pandemic, most elderly people are in social isolation, which could be a "serious public health concern" because of their heightened risk of cardiovascular, autoimmune, neurocognitive, and mental health problems (23). Moreover, recent studies have shown that social disconnection puts elderly people at greater risk of depression and anxiety (24)but less is known about the distinct contributions of different aspects of isolation. We aimed to distinguish the pathways through which social disconnectedness (eg, small social network, infrequent social interaction. As observed in the current study, the elevated anxiety and stress levels among elderly patients with TMDs might be related to their social disconnection due to the ongoing COVID-19 pandemic; however, further comparative studies with long-term results are necessary to determine the exact relation.

This study has clearly shown that bruxism is the main condition, which might be correlated to elevated anxiety and stress levels and requires multidisciplinary management. Many patients suffering from bruxism are also primarily seeking treatment from neurologists and/or psychiatrists. In the management of bruxism, selective serotonin reuptake inhibitors (SSRIs) could be a feasible option to overcome elevated stress and anxiety levels. However, at this point, a well-described (still underreported) condition should be highlighted. SSRI-associated bruxism, which could occur as an adverse reaction to antidepressant therapy, is a rare entity which neurologists, psychiatrists, and dentists should be aware of. Besides, increased blood pressure and sleep-related bruxism warrant further research; thus, the relationship between sleep apnoea and hypertension has not yet been well documented. Considering that systemic diseases and medication needs increase with age, dentists should properly review the medication of elderly patients with TMDs.

One might suggest that complete denture wearers are more likely to develop TMD symptoms; however, no robust association between prosthetic factors and TMD was found in the literature (25). Because of the controversies surrounding the asso- 
ciation between TMD symptoms and edentulism, complete denture wearers were excluded from the study.

Patients with both intra- and extra-articular TMJ pathologies presented with elevated depression and anxiety levels, whereas those with bruxism, disc displacement without reduction, and myofascial

\section{REFERENCES}

1. Scrivani SJ, Keith DA, Kaban LB. Temporomandibular disorders. N Engl J Med. 2008;359(25):2693-705. (PMID: 19092154)

2. Durham J, Newton-John TRO, Zakrzewska JM. Temporomandibular disorders. BMJ. 2015;350:h1154. (PMID: 25767130)

3. Manfredini D, Guarda-Nardini L, Winocur E, Piccotti F, Ahlberg J, Lobbezoo F. Research diagnostic criteria for temporomandibular disorders: a systematic review of axis I epidemiologic findings. Oral Surg Oral Med Oral Pathol Oral Radiol Endod. 2011;112(4):453-62. (PMID: 21835653)

4. Yadav S, Yang Y, Dutra EH, Robinson JL, Wadhwa S. Temporomandibular Joint Disorders in Older Adults. J Am Geriatr Soc. 2018;66(6):1213-7. (PMID: 29719041)

5. Ogura I, Kaneda T, Mori S, Sakayanagi M, Kato M. Magnetic resonance characteristics of temporomandibular joint disc displacement in elderly patients. Dento Maxillo Facial Radiol. 2012;41(2):122-5. (PMID: 22116131)

6. Calixtre LB, Grüninger BL da S, Chaves TC, Oliveira $A B$ de. Is there an association between anxiety/depression and temporomandibular disorders in college students? J Appl Oral Sci Rev FOB. 2014;22(1):15-21. (PMID: 24626244)

7. Schiffman E, Ohrbach R, Truelove E et al. Diagnostic Criteria for Temporomandibular Disorders (DC/ TMD) for Clinical and Research Applications: recommendations of the International RDC/TMD Consortium Network* and Orofacial Pain Special Interest Groupt. J Oral Facial Pain Headache. 2014;28(1):627. (PMID: 24482784)

8. Fillingim RB, Ohrbach R, Greenspan JD et al. Psychological factors associated with development of pain syndrome showed significantly higher anxiety and depression levels.

The literature is remarkably deficient in the evaluation of the relationship between TMD and the undiagnosed psychological status of a patient. Despite the limitations, the current study has confirmed that this relation warrants further research.

TMD: the OPPERA prospective cohort study. J Pain. 2013;14(12 Suppl):T75-90. (PMID: 24275225)

9. Beck AT, Ward CH, Mendelson M, Mock J, Erbaugh J. An inventory for measuring depression. Arch Gen Psychiatry. 1961;4:561-71. (PMID: 13688369)

10. Beck AT, Epstein N, Brown G, Steer RA. An inventory for measuring clinical anxiety: psychometric properties. J Consult Clin Psychol. 1988;56(6):893-7. (PMID: 3204199)

11. Dreiman BB. Thoughts on the psychological component of TMJ/TMD syndrome. Oral Surg Oral Med Oral Pathol Oral Radiol Endod. 2011;112(5):555-6. (PMID: 21889373)

12. Kinney RK, Gatchel RJ, Ellis E, Holt C. Major psychological disorders in chronic TMD patients: implications for successful management. J Am Dent Assoc. 1992;123(10):49-54. (PMID: 1401593)

13. Chuinsiri N, Jitprasertwong P. Prevalence of self-reported pain-related temporomandibular disorders and association with psychological distress in a dental clinic setting. J Int Med Res. 2020;48(9):300060520951744. (PMID: 32883120)

14. Saeed T, Riaz N. Level of Depression in Temporomandibular Disorder Patients. J Pak Dent Assoc. 2018;27(03):100-5. (DOI:10.25301/JPDA.273.100)

15. Bertoli E, de Leeuw R. Prevalence of Suicidal Ideation, Depression, and Anxiety in Chronic Temporomandibular Disorder Patients. J Oral Facial Pain Headache. 2016;30(4):296-301. (PMID: 27792796)

16. Resende CMBM de, Rocha LGD da S, Paiva RP de et al. Relationship between anxiety, quality of life, and sociodemographic characteristics and temporomandibular disorder. Oral Surg Oral Med Oral Pathol Oral Radiol. 2020;129(2):125-32. (PMID: 31784398)

17. Resende CMBM de, Alves AC de M, Coelho LT, Alchieri JC, Roncalli AG, Barbosa GAS. Quality of life 
and general health in patients with temporomandibular disorders. Braz Oral Res. 2013;27(2):116-21. (PMID: 23459771)

18. Braga SP, Fiamengui LMSP, da Silveira VRS et al. Insights for temporomandibular disorders management: From psychosocial factors to genetics-A case report. Spec Care Dentist. 202;41(1):85-91. (PMID: 33152157)

19. Machado NAG, Costa YM, Quevedo HM et al. The association of self-reported awake bruxism with anxiety, depression, pain threshold at pressure, pain vigilance, and quality of life in patients undergoing orthodontic treatment. J Appl Oral Sci. 2020;28:e20190407. (PMID: 32236355)

20. Maślak-Bereś M, Loster JE, Wieczorek A, Loster BW. Evaluation of the psychoemotional status of young adults with symptoms of temporomandibular disorders. Brain Behav. 2019;9(11):e01443. (PMID: 31613063)

21. Kandola A, Lewis G, Osborn DPJ, Stubbs B, Hayes JF. Depressive symptoms and objectively measured physical activity and sedentary behaviour throughout adolescence: a prospective cohort study. Lancet Psychiatry. 2020;7(3):262-71. (PMID: 32059797)

22. Kmeid E, Nacouzi M, Hallit S, Rohayem Z. Prevalence of temporomandibular joint disorder in the Lebanese population, and its association with depression, anxiety, and stress. Head Face Med. 2020;16(1):19. (PMID: 32887633)

23. Armitage R, Nellums LB. COVID-19 and the consequences of isolating the elderly. Lancet Public Health. 2020;5(5):e256. (PMID: 32199471)

24. Santini ZI, Jose PE, York Cornwell E et al. Social disconnectedness, perceived isolation, and symptoms of depression and anxiety among older Americans (NSHAP): a longitudinal mediation analysis. Lancet Public Health. 2020;5(1):e62-70. (PMID: 31910981)

25. Ribeiro JAM, de Resende CMBM, Lopes ALC, Farias-Neto A, Carreiro A da FP. Association between prosthetic factors and temporomandibular disorders in complete denture wearers. Gerodontology. 2014;31(4):308-13. (PMID: 23448239) 